\title{
ON UNIFORMLY AMENABLE GROUPS
}

\author{
JANUSZ WYSOCZÁNSKI
}

\begin{abstract}
We consider the conditions of the uniform amenability of discrete groups. The equivalence of the uniform Følner and Kesten conditions is proved. Also an example of the group which is amenable but not uniformly amenable is given.
\end{abstract}

1. Introduction. There are many well-known characterizations of amenable groups. We consider in this paper two of them given by E. Følner [2] and H. Kesten [9]. The relationships between these conditions of amenability were considered by V. A. Kaimanovich and V. M. Vershik [6] in the context of random walks on groups.

Some properties related to amenability for discrete groups were introduced by M. Bożejko [1] and G. Keller [7]. They called the discrete amenable group $G$ uniformly amenable if there exists a function $a:(0,1) \times N \rightarrow N$ such that for every finite subset $A$ of the group $G$ and every $e \in(0,1)$ there is a finite subset $U$ of $G$ such that

1. $|U| \leq a(e,|A|)=a_{e}(|A|)$, and

2. $|A U|<(1+e)|U|$.

Here $|A|$ is the cardinality of $A$ and $A U=\{a \cdot u: a \in A, u \in U\}$. The above condition will be called the uniform Følner condition for $G(\mathrm{UF}(G))$. Keller proved that a group $G$ is uniformly amenable if and only if any nonstandard model $G^{*}$ of $G$ is amenable. He used methods of nonstandard analysis. These methods are not widely known and accepted. In this paper we give a simple combinatorial proof of Keller's theorem (Lemmas 2 and 4) for ultrapowers of $G$. In fact, it follows that a discrete group is uniformly amenable if and only if its ultrapower is uniformly amenable.

M. Bożejko proposed to find an analogous uniform condition for the Kesten condition $(\mathrm{K}(G))$ :

For every finite symmetric subset $A$ of the group $G$,

$$
\lim _{n}\left(m_{2 n}(e)\right)^{1 / 2 n}=|A|,
$$

where $m_{2 n}$ is the $2 n$th convolution power of the characteristic function $m$ of $A$. We will consider the uniform convergence in $(*)$ with respect to the cardinality of $A$. This will be called the uniform condition of Kesten $(\mathrm{UK}(G))$. We prove in this paper that the uniform Følner and Kesten conditions are equivalent.

II. Ultrapowers. The filter $F$ on the natural numbers $N$ is the family of subsets $A, B, \ldots$ of $N$ satisfying the following conditions:

1. $A, B \in F$ implies $A \cap B \in F$.

Received by the editors November 18, 1986.

1980 Mathematics Subject Classification (1985 Revision). Primary 43A07.

Key words and phrases. Amenable groups, uniform amenability, ultrapowers of groups. 
2. $A \in F, A \subset C$ for some $C \subset N$ implies $C \in F$.

If we denote by $F$ the Fréchet filter consisting of cofinite subsets of $N$, then there exists a maximal filter $w$ on $N$ containing $F$. This filter $w$ is called an ultrafilter and has a maximal property such that for all $A \subset N$

$$
A \in W \quad \text { if and only if } \quad N-A \notin w .
$$

From this it follows that if $E \in w$ and $E$ is the union of some disjoint sets $V_{1}, \ldots, V_{n}$, then exactly one of them is an element of $w$.

Let $G$ be a countable discrete group. One defines the ultrapower $G^{*}$ of $G$ with respect to $w$. If $G^{N}$ denotes the set consisting of sequences $\left(g_{n}\right)_{n=1}^{\infty}, g_{n} \in G$, for all $n \in N$, then $G^{N}$ is a group with multiplication defined by

$$
\left(g_{n}\right) \cdot\left(\tilde{g}_{n}\right)=\left(g_{n} \cdot \tilde{g}_{n}\right) .
$$

Its subgroup $I_{w}=\left\{\left(g_{n}\right):\left\{n: g_{n}=e\right\} \in w\right\}$ is normal so one gets that the quotient $G^{*}=G^{N} / I_{w}$ is a group. $G$ can be regarded as the subgroup of $G^{*}$ consisting of constant (modulo $I_{w}$ ) sequences.

If we take a finite subset $A^{*}$ of $G^{*}$, then its elements can be represented by sequences $\left(g_{n}^{1}\right),\left(g_{n}^{2}\right), \ldots$ Let us assume that $\left|A^{*}\right|=k$; then we can consider the matrix $\left(g_{n}^{j}\right)_{n=1}^{\infty},{ }_{j=1}^{k}$ whose columns are sequences $\left(g_{n}^{1}, g_{n}^{2}, \ldots, g_{n}^{k}\right)$. One can choose the representing sequences in such a way that

$$
\left\{n: \operatorname{card}\left\{g_{n}^{1}, \ldots, g_{n}^{k}\right\}=k\right\} \in w .
$$

This follows by the induction on $k$ :

Assume that we chose $\left(g_{n}^{1}\right), \ldots,\left(g_{n}^{k-1}\right)$ and denote

$$
\begin{aligned}
E & =\left\{n: \operatorname{card}\left\{g_{n}^{1}, \ldots, g_{n}^{k-1}\right\}=k-1\right\}, \\
E_{j} & =\left\{n \in E: g_{n}^{j}=g_{n}^{k}\right\} .
\end{aligned}
$$

The sets $E_{j}$ are disjoint. If their union belongs to $w$, then exactly one of them (for example $\left.E_{1}\right)$ is in $w$. Therefore, $\left(g_{n}^{k}\right)$ and $\left(g_{n}^{1}\right)$ must represent the same element of $A^{*}$. But this is not true. Thus, $E_{k}=E-\left(E_{1} \cup \cdots \cup E_{k-1}\right)$ belongs to $w$. This means that $g_{n}^{k} \neq g_{n}^{j}$ for all $n \in E_{k}$ and $j=1, \ldots, k-1$. We get

Proposition 1. Any finite subset $A^{*}$ of $G^{*}$ is an ultraproduct of the sequence of finite subsets $A_{n}$ of $G$ with the same cardinality as $A^{*}$.

On the other hand, if we take different sets $A_{n} \subset G$ with $\left|A_{n}\right|=k$ for all $n \in N$, then their ultraproduct $A^{*}$, defined as

$$
A^{*}=\left(\prod_{n} A_{n}\right) / I_{w},
$$

is a subset of $G^{*}$ consisting exactly of $k$ elements.

III. Theorem. Recall that we consider the ultrapower $G^{*}$ of the discrete group $G$ with respect to the ultrafilter $w$, on the set $N$ of natural numbers, containing the filter of cofinite subsets of $N$. 
LEMMA 1. $\mathrm{UK}(G)$ implies $\mathrm{K}\left(G^{*}\right)$.

PrOOF. Let us consider the subset $A^{*}$ of the group $G^{*}$ whose cardinality is $k$, and assume that $A^{*}$ is an ultrapower of a sequence $\left(A_{n}\right)$. Denote by $v_{n}$ (respectively $v$ ) the characteristic function of the set $A_{n}$ (respectively $A$ ), and by $v_{n}^{* r}$ the $r$ th convolution power of $v_{n}$. We can write

$$
v_{n}^{* r}(e)=\sum_{x_{i_{1}}, \ldots, x_{i_{r}}} d_{x_{i_{1}} \cdots x_{i_{r}}}(c), \quad r \text {-even }
$$

where

$$
d_{x}(e)= \begin{cases}1, & x=e, \\ 0, & x \neq e .\end{cases}
$$

Each summand in $(* *)$ is equal to 0 or 1 ; thus, for every $r$ we can represent $v_{n}^{* r}(e)$ as a sequence

$$
t_{n}(r)=\left(s_{1}, \ldots, s_{k^{r}}\right)
$$

with $s_{j} \in\{0,1\}$. For fixed $r \in N$ there is only a finite number of such sequences, so we can decompose $N$ into a finite union of disjoint sets $E_{1}, \ldots, E_{l}$ for which $n, k \in E_{j}$ if and only if $t_{n}(r)=t_{k}(r)$. The maximal property of $w$ implies that exactly one of $E_{j}$ 's belongs to $w$. Therefore, the sequence

$$
t(r)=\left(s_{1}, \ldots, s_{k^{r}}\right),
$$

which corresponds to this set, is a representation of $A^{*}$ and

$$
v^{* r}(e)=\sum_{j=1}^{k^{r}} s_{j}
$$

The lemma now follows.

LEMMA 2. $\mathrm{F}\left(G^{*}\right)$ implies $\mathrm{UF}(G)$.

ProOF. Let us consider a sequence $\left(A_{n}\right), A_{n} \subset G,\left|A_{n}\right|=k$, and let $\left(U_{n}\right)$ be the sequence whose elements are the Følner sets for $A_{n}$ with minimal cardinality for fixed $e$ :

1. $\left|A_{n} \cdot U_{n}\right|<(1+e)\left|U_{n}\right|$

2. if $\left|A_{n} \cdot \tilde{U}_{n}\right|<(1+e)\left|\tilde{U}_{n}\right|$, then $\left|U_{n}\right| \leq\left|\tilde{U}_{n}\right|$.

Note that $G$ as a subgroup of $G^{*}$ is amenable. Assume that

$$
\lim _{n}\left|U_{n}\right|=\infty
$$

and denote by $A^{*}$ the ultraproduct of the sets $A_{n}$. Amenability of $G^{*}$ implies that there exists a Følner set $U^{*}$ for $A^{*}$. This set is an ultraproduct of subsets $\tilde{U}_{n}$ of $G$ with $\left|\tilde{U}_{n}\right|=\left|U^{*}\right|$. Thus $\tilde{U}_{n}$ is a Følner set for $A_{n}$ where $n \in E$ and $E \in w$. So we obtain

$$
\left|U_{n}\right| \leq\left|\tilde{U}_{n}\right|=\left|U^{*}\right| \text { for } n \in E,
$$

which is impossible because of our assumption. Therefore, $G$ is uniformly amenable. 
LEMMA 3. $\mathrm{UF}(G)$ implies $\mathrm{UK}(G)$.

This is an easy consequence of Kaimanovich's Theorem 3 [5]: For every $n \in N$, $0<e<1,0<h<1$,

$$
m_{2 n}(e) \geq(1-h)^{n} \cdot \frac{1-2 e / h^{2}}{U_{e}},
$$

where $U_{e}$ is a Følner set for $e$ and $\operatorname{supp} m$.

REMARK. In fact, it follows that $\mathrm{UF}(G)$ implies

$$
\left(m_{2 n}(e)\right)^{1 / 2 n} \rightarrow|\operatorname{supp} m|
$$

uniformly with respect to all measures with support of the same cardinality.

Now we can prove the main

THEOREM. Let $G$ be a discrete group; then $G$ satisfies the uniform Følner condition if and only if $G$ satisfies the uniform Kesten condition.

Proof. Let us assume $\operatorname{UK}(G)$; then by Lemma $1, G^{*}$ is amenable, and by Lemma 2, the uniform Følner condition for $G$ holds. The opposite implication is by Lemma 3 . We call the group $G$ uniformly amenable if one of the two conditions holds for $G$.

To give a simple proof of Keller's theorem we need the following

LEMMA 4. $\mathrm{UF}(G)$ implies $\mathrm{F}\left(G^{*}\right)$.

Proof. Let us consider a subset $A^{*}$ in $G^{*}$ of cardinality $k$, which is an ultraproduct of some sets $A_{n} \subset G$ of cardinality $k$. By the assumption we can find a sequence $\left(U_{n}\right)$ of $\mathrm{F} \varnothing \operatorname{lner}$ sets for $A_{n}$ :

$$
\left|A_{n} \cdot U_{n}\right|<(1+e)\left|U_{n}\right|, \quad\left|U_{n}\right| \leq M
$$

$e$ is fixed in $(0,1)$. We numerate elements in $A_{n}$ and $U_{n}$ :

$$
A_{n}=\left\{a_{1 n}, \ldots, a_{k n}\right\}, \quad U_{n}=\left\{u_{1 n}, u_{2 n}, \ldots\right\} .
$$

Let us take two matrices $\left(a_{i j}\right)_{i=1}^{k}, \underset{j=1}{\infty},\left(u_{i j}\right)_{i=1}^{M}, \underset{j=1}{\infty}$ whose columns are sets $A_{n}$ and $U_{n}$, respectively. If $\left|U_{n}\right|<M$, we can repeat one element of $U_{n}$ to fulfill the $n$th column. Denote by $V_{r}, r=1,2, \ldots$, the sets with the following property: for every $n, k \in V_{r}, a_{i n} \cdot u_{j n}=a_{s n} \cdot u_{t n}$ if and only if $a_{i k} \cdot u_{j k}=a_{s k} \cdot u_{t k}$, where $1 \leq i, s \leq k, 1 \leq j, t \leq M$.

These sets are disjoint and their union is the set $N$ of all natural numbers. It follows that there is only a finite number (not greater than $k \cdot M$ ) of different $V_{r}$ 's. So exactly one of them is an element of ultrafilter $w$. Therefore,

$$
\left|A^{*} \cdot U^{*}\right| \leq(1+e) \cdot M
$$

so $G$ is amenable (even uniformly!).

IV. The example. We give an example of an amenable, but not uniformly amenable, group. Let $G_{p}$ be the group of $3 \times 3$ matrices of the form

$$
\left(\begin{array}{lll}
1 & x & z \\
0 & 1 & y \\
0 & 0 & 1
\end{array}\right)
$$


where $x, y, z \in Z_{p}, p$ is prime, and $Z_{p}=\{0,1, \ldots, p-1\}$ denotes the finite field with the addition and multiplication modulo $p$. Taking two matrices

$$
a=\left(\begin{array}{lll}
1 & 1 & 0 \\
0 & 1 & 0 \\
0 & 0 & 1
\end{array}\right), \quad b=\left(\begin{array}{lll}
1 & 0 & 0 \\
0 & 1 & 1 \\
0 & 0 & 1
\end{array}\right)
$$

and the set $A_{p}=\left\{a, a^{-1}, b, b^{-1}\right\}$, we try to estimate $m_{2 p}(e)\left(m_{2 p}\right.$ is the $2 p$ th convolution power of the characteristic function $m$ of the set $A_{p}$ ). To do this we need to estimate the number of possibilities that the element

$$
e=\left(\begin{array}{lll}
1 & 0 & 0 \\
0 & 1 & 0 \\
0 & 0 & 1
\end{array}\right)
$$

can be written as the product

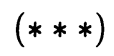

$$
e=x_{i_{1}} \cdots x_{i_{2 p}}
$$

with $x_{i_{1}}, \ldots, x_{i_{2 p}} \in A_{p}$. If $a$ (respectively $a^{-1}, b, b^{-1}$ ) occurs in the product $(* * *) n_{1}$ (respectively $n_{2}, m_{1}, m_{2}$ ) times, then it necessarily must be (respectively $\left.n_{2}, m_{1}, m_{2}\right)$ times, then it necessarily must be

(i) $n_{1} \equiv n_{2}(\bmod p)$,

(ii) $m_{1} \equiv m_{2}(\bmod p)$, and

(iii) $n_{1}+n_{2}+m_{1}+m_{2}=2 p$.

This follows because the considered product is the matrix of the form

$$
\left(\begin{array}{ccccc}
1 & n_{1}+p-1 & n_{2} & z & \\
0 & 1 & m_{1}+p-1 & m_{2} \\
0 & 0 & 1 &
\end{array}\right) .
$$

Condition (i) is equivalent to

$$
n_{1}=n_{2} \quad \text { or } \quad\left|n_{1}-n_{2}\right|=p \quad \text { or } \quad\left|n_{1}-n_{2}\right|=2 p .
$$

Similarily, (ii) is equivalent to

$$
m_{1}=m_{2} \quad \text { or } \quad\left|m_{1}-m_{2}\right|=p \quad \text { or } \quad\left|m_{1}-m_{2}\right|=2 p .
$$

If $\left|n_{1}-n_{2}\right|=2 p$, then by (iii) $n_{1}=2 p, n_{2}=m_{1}=m_{2}=0$ or $n_{2}=2 p$, $n_{1}=m_{1}=m_{2}=0$; similarily in the case $\left|m_{1}-m_{2}\right|=2 p$. This gives at most four representations of $e$ :

$$
a^{2 p}=e, \quad a^{-2 p}=e, \quad b^{2 p}=e, \quad b^{-2 p}=e .
$$

If $\left|n_{1}-n_{2}\right|=p$, then $n_{1}=n_{2}+p$ or $n_{2}=n_{1}+p$. We consider only the first case: $n_{1}=n_{2}+p$. Then

$$
\begin{aligned}
2 n_{2}+p+m_{1}+m_{2} & =2 p \\
2 n_{2}+m_{1}+m_{2} & =p .
\end{aligned}
$$

Now if $\left|m_{1}-m_{2}\right|=p$, then $2 n_{2}+2 m_{1}=0$ or $2 n_{2}+2 m_{2}=0$, which is impossible for $n_{2}>0$. If $n_{2}=0$, then we obtain

$$
m_{1}+m_{2}=p \text { and }\left|m_{1}-m_{2}\right|=p
$$


which has two solutions: $m_{1}=p, m_{2}=0$ and $m_{1}=0, m_{2}=p$. In these cases the corresponding representations of $e$ contain $p$ times $a$ (or $a^{-}$) and $p$ times $b$ (or $\left.b^{-1}\right)$. One can see that there are at most $4\left(\begin{array}{c}2 p \\ p\end{array}\right)$ such representations. The case $\left|n_{1}-n_{2}\right|=p, m_{1}=m_{2}$ is impossible because $p$ is prime. The last case is when $n_{1}=n_{2}=n, m_{1}=m_{2}=m, n+m=p$. For every $n, 1 \leq n \leq p-1$, we have $\left(\begin{array}{c}2 p \\ p\end{array}\right) \cdot\left(\begin{array}{c}2 p-n \\ n\end{array}\right) \cdot\left(\begin{array}{c}2 p-2 n \\ p-n\end{array}\right)$ representations of $e$ of the form $(* * *)$ in which $a$ and $a^{-1}$ occur $n$ times, and $b$ and $b^{-1}$ occur $p-n$ times. So we obtain

$$
\begin{aligned}
m_{2 p}(e) & \leq 4+4\left(\begin{array}{c}
2 p \\
p
\end{array}\right)+\sum_{n=1}^{p-1}\left(\begin{array}{c}
2 p \\
p
\end{array}\right) \cdot\left(\begin{array}{c}
2 p-n \\
n
\end{array}\right) \cdot\left(\begin{array}{c}
2 p-2 n \\
p-n
\end{array}\right) \\
& =4+4\left(\begin{array}{c}
2 p \\
p
\end{array}\right)+\sum_{n=1}^{p-1} 2^{p}\left(\begin{array}{l}
p \\
n
\end{array}\right)^{2} \\
& \leq 2^{p+1}\left(\begin{array}{c}
2 p \\
p
\end{array}\right) .
\end{aligned}
$$

Thus, we obtain

$$
\lim _{p}\left(m_{2 p}(e)\right)^{1 / 2 p} \leq \sqrt{8}
$$

On the other hand, $G_{p}$ is an amenable group, so

$$
\lim _{r}\left(m_{2 r}(e)\right)^{1 / 2 r}=4 \text {. }
$$

Therefore, if we define $G=\bigoplus_{p \text { prime }} G_{p}$, then we have that $G$ is amenable but not uniformly amenable because there is a sequence $\left(A_{p}\right)$ of subsets of $G$ with $\left|A_{p}\right|=4$ for which the uniform Kesten condition does not hold.

The author would like to thank Marek Bożejko for helpful discussions and proposing the subject of this paper.

\section{REFERENCES}

1. M. Bożejko, Uniformly amenable discrete groups, Math. Ann. 251 (1980), 1-6.

2. E. Følner, On groups with full Banach mean value, Math. Scand. 3 (1955), 243-254.

3. F. Greenleaf, Invariant means on topological groups and their applications, Van NostrandRheinhold, New York, 1969.

4. S. Heinrich, Ultraproducts in Banach space theory, J. Reine Angew. Math. 313 (1980), 72-104.

5. V. A. Kaimanovich, The spectral measure of transition operator and harmonic functions connected with the random walks on discrete groups, LOMI, Leningrad, 1980.

6. V. A. Kaimanovich and A. M. Vershik, Random walks on discrete groups: boundary and entropy, Ann. Probab. 11 (1983), 457-490.

7. G. Keller, Amenable groups and varieties of groups, Illinois J. Math. 16 (1972), 257-268.

8. H. Kesten, Symmetric random walks on groups, Trans. Amer. Math. Soc. 92 (1959), 336-354.

9. __ Full Banach mean values on countable groups, Math. Scand. 7 (1959), 146-156.

Institute of Mathematics, Wroclaw University, PL. GRUNWALDZKi 2/4, 50-384 WROCLAW, POLAND 